\title{
A cone programming approach to the bilinear matrix inequality problem and its geometry ${ }^{1}$
}

\author{
Mehran Mesbahi ${ }^{a, *}$, George P. Papavassilopoulos ${ }^{b, 2}$ \\ a Jet Propulsion Laboratory, California Institute of Technology, 4800 Oak Grove Drive, \\ Pasadena, CA 91109, USA \\ ' Department of Electrical Engineering - Systems, University of Southern California, \\ Los Angeles, CA 90089-2563, USA
}

Received 10 January 1995

\begin{abstract}
We discuss an approach for solving the Bilinear Matrix Inequality (BMI) based on its connections with certain problems defined over matrix cones. These problems are, among others, the cone generalization of the linear programming (LP) and the linear complementarity problem (LCP) (referred to as the Cone-LP and the Cone-LCP, respectively). Specifically, we show that solving a given BMI is equivalent to examining the solution set of a suitably constructed Cone-LP or Cone-LCP. This approach facilitates our understanding of the geometry of the BMI and opens up new avenues for the development of the computational procedures for its solution. (C) 1997 The Mathematical Programming Society, Inc. Published by Elsevier Science B.V.
\end{abstract}

Keywords: Bilinear matrix inequalities; Linear complementarity problem over cones; Linear programming over cones; Robust control

\section{Introduction}

The Bilinear Matrix Inequality (BMI) is considered to be the central problem in the field of robust control. The BMI feasibility problem is as follows: Given symmetric matrices $H_{i j} \in \mathbb{R}^{p \times p}(i=1, \ldots, n, j=1, \ldots m)$, does there exist $x \in \mathbb{R}^{n}$, and $y \in \mathbb{R}^{m}$, such that $\sum_{i=1}^{n} \sum_{j=1}^{m} x_{i} y_{j} H_{i j}$ is positive definite. As it was shown by Safonov et al. [19]

\footnotetext{
* Corresponding author. E-mail: mesbahi@hafez.jpl.nasa.gov.

' Research supported in part by the National Science Foundation under Grant CCR-9222734.

2E-mail: yorgos@bode.usc.edu.
} 
it is possible to reduce a wide array of control synthesis problems, such as the fixedorder $H^{\infty}$ control, $\mu / k_{m}$-synthesis, decentralized control, robust gain-scheduling, and simultaneous stabilization to a BMI. It is also known that the Linear Matrix Inequality (LMI) approach to control synthesis [3] is a special case of the BMI. Since the LMI is equivalent to the Semi-Definite Programming Problem (SDP), the BMI can also be considered as a generalization of the SDP. It is therefore not surprising that the solution to the BMI is not only of central importance in the context of robust control [20], but also in its connections with the SDPs and the LMIs.

The BMI can be reformulated as a nonconvex programming problem. More specifically, Safonov and Papavassilopoulos [20] have shown that the BMI feasibility problem is equivalent to checking whether the diameter of a certain convex set is greater than two. Since this is equivalent to a maximization of a convex function subjected to a set of convex constraints (an NP-hard problem), no efficient algorithm is believed to exist for a general BMI. Moreover, Toker and Özbay have recently shown that the BMI feasibility is an NP-hard problem by reducing the Subset-Sum problem to it [22].

The computational procedures which have been suggested for solving the BMI rely on a global optimization approach [5]. There are at least three issues which have to be addressed in connection with the BMI and the global optimization methods:

(1) What are the geometric interpretations of the BMI?

(2) What are the specific properties of the global optimization problem which arises from the BMI, and whether these properties can be used to devise more efficient algorithms for the BMI?

(3) Which instances of the BMI can be solved efficiently? Moreover, are there instances for which certain "structural" properties can be established, for example, the convexity of the solution set?

All of the above issues can be addressed by studying the BMI on its own. Nevertheless, we believe that many important structural and computational issues of the BMI can be studied by establishing a connection between the BMI and the problems which are more well-understood in the optimization theory.

We recall that the SDP, is a special instance of the linear programming problem (LP) over a matrix cone (Cone-LP), namely the cone of the symmetric positive semi-definite matrices. In fact this observation is exactly the motivation for the generalization of the interior point methods for the LP to solve instances of the SDP $[1,17]$. The question then arises as to whether the BMI could be transformed to a problem which is almost a Cone-LP (we say almost, since as it was mentioned previously, the BMI is a nonconvex optimization problem and in general, cannot possibly be transformed to a convex one). This approach is discussed further in Sections 3.3 and 3.4.

We also recognize that the BMI is a generalization of the SDP with a nonconvex feature (in fact, it is a so-called biconvex programming problem). The LP, for which the SDP is a generalization of, has as its close "relative", the Linear Complementarity problem (LCP) [4]. Stated concisely, the LCP can be formulated as follows: Given $M \in \mathbb{R}^{n \times n}$ and $q \in \mathbb{R}^{n}$, find $z \in \mathbb{R}^{n}$ (if it exists), such that 


$$
\begin{aligned}
& z \geqslant 0 \\
& q+M z \geqslant 0 \\
& z^{\prime}(q+M z)=0
\end{aligned}
$$

where " $z$ " denotes the transpose of the vector $z$, and the ordering " $\geqslant$ " for vectors is interpreted component-wise. In the LCP theory, there is a very fine classification of the complementarity problems, many of which correspond to nonconvex optimization. More specifically, although the general LCP is an NP-hard problem, an immense amount of insight has been provided by studying various matrix classes that arise in the LCP formulation. These "insights" are both structural (e.g., existence and uniqueness of the solution, convexity of the solution set, etc.), and algorithmic (e.g., Lemke's algorithm for the bimatrix game problems, etc.). We believe that the same approach can be adopted for the BMI. In fact, one might even suspect that the BMI can be transformed to a cone generalization of the LCP (Cone-LCP), by which, some relevant results from the complementarity theory can be employed to address certain structural, and possibly, algorithmic, issues of the BMI. We discuss this possibility in Section 3.5. Nevertheless, we should keep in mind that even the LCP (without the cone generalization, which is of our prime interest in this paper) is in general a difficult computational problem. However, the complementarity approach facilitates our understanding of certain structural issues, and at the same time, it provides an avenue for recognizing efficiently solvable instances, of the BMI. In fact, as we show, the Cone-LCP that comes up in our study of the BMI, has a linear operator which is copositive with respect to the cone of positive semi-definite matrices (a term to be defined in Section 2).

The relationship among the various problems that are considered in this paper can be illustrated as in Fig. 1. In this figure, the vertical arrows are used to indicate the "cone" generalizations of the problems, and the horizontal arrows to indicate that the problem formulation on the head side of the arrow, has as its special case, the problem on the tail side. In Fig. 1, the question marks between the BMI and the Cone-LP, and the Cone-LCP, are the main issues that are considered in this paper. In particular, our main results indicate that solving a given BMI is equivalent to examining the solution set of a suitably constructed Cone-LP or Cone-LCP.

An intermediate step in adopting a Cone-LP and a Cone-LCP approach for solving the BMI, is the introduction of a problem which we shall refer to as the Extreme Form Problem (EFP). Formulated in the $n$-dimensional Euclidean space $\mathbb{R}^{n}$, and denoting the

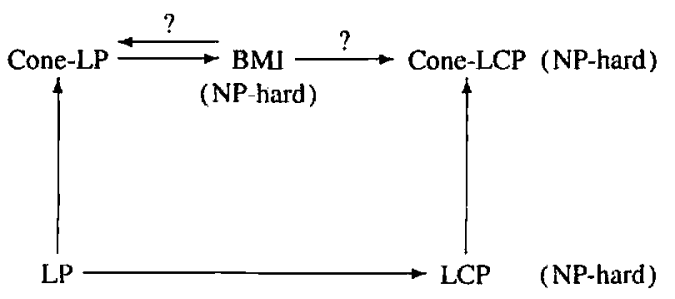

Fig. I. Ermbeddings and cone generalizations of various problems. 
nonnegative orthant by $\mathbb{R}_{+}^{n}$, the EFP has the following formulation: Given $M: \mathbb{R}^{n} \rightarrow \mathbb{R}^{n}$, find $z \in \mathbb{R}^{n}$ (if it exists), such that,

$$
z \geqslant 0 \text {, }
$$

$M z>0$,

$z$ is an extreme form of $\mathbb{R}_{+}^{n}$.

An extreme form (or an extreme ray) of a cone is a face of the cone which is a half-line emanating from the origin $[8,18]$. The above instance of the EFP is referred to as the $\operatorname{EFP}_{\mathbb{R}^{\prime \prime}}(M)$. The EFP, as we just defined, is not an interesting problem. In fact, the $\operatorname{EFP}_{\mathbb{R}_{+}^{\prime \prime}}(M)$ has a solution if and only if $M$ has a positive column. On the other hand, the EFP becomes non-trivial when $\mathbb{R}_{+}^{n}$ is replaced by an arbitrary cone. We have used the cone generalization of the EFP as an intermediate step in the Cone-LP/LCP approach for solving the BMI. It can be argued that computational procedures could be developed for the EFP directly, without formulating it as a Cone-LP or a Cone-LCP. We have chosen this approach, since at the present time, the Cone-LP and the Cone-LCP seem to be more amenable for the application of the interior point methods than the EFP. It is still an open question whether an interior point method can be adapted for solving the EFPs directly.

The organization of this paper is as follows. In the next section we present some basic definitions, certain matrix cones, as well as the precise formulation of the Cone-LP, the Cone-LCP, and the EFP. In the same section a glossary of notations that are used in the paper is provided. In Section 3, the "cone" formulations of the BMI are presented. We also discuss certain computational implications of these reductions. In the final section, we discuss aspects of the problem that call for further investigations.

\section{Preliminaries}

In this section we define the Cone-LP, the Cone-LCP, and introduce the Extreme Form Problem (EFP) over finite dimensional cones. We also mention certain matrix classes, which when generalized appropriately, will make the transformation of the BMI to the EFP and the Cone-LP/LCP more explicit.

\subsection{The Cone-LP, the Cone-LCP, and the EFP}

Prior to defining the Cone-LP, the Cone-LCP, and the EFP, few basic definitions are in order. The definitions include those of a cone, the dual cone of a set, and the notion of positivity and copositivity of a linear map with respect to a given cone. We shall restrict ourselves to the finite dimensional vector spaces in all subsequent sections.

Let $\mathcal{H}$ be a finite dimensional Hilbert space equipped with the inner product $\langle\cdot, \cdot\rangle$ : $\mathcal{H} \times \mathcal{H} \rightarrow \mathbb{R}$ (e.g., the $n$-dimensional Euclidean space or the space of $n \times n$ matrices, with the appropriate notion of an inner product defined on them). A set $\mathcal{K} \subseteq \mathcal{H}$ is a 
cone if for all $\alpha \geqslant 0, \alpha \mathcal{K} \subseteq \mathcal{K}$. $\mathcal{K}$ is a convex cone, if $\mathcal{K}$ is a cone and it is convex, i.e., for all $\alpha \in[0,1], \alpha \mathcal{K}+(1-\alpha) \mathcal{K} \subseteq \mathcal{K}$, or equivalently, if $\mathcal{K}$ is a cone and $\mathcal{K}+\mathcal{K} \subseteq \mathcal{K}$. A convex cone $\mathcal{K}$ is called pointed if $\mathcal{K} \cap(-\mathcal{K})=\{0\}$, and solid if it has a non-empty interior. An extreme form (or an extreme ray) of a convex cone $\mathcal{K}$ is a subset $E=\{\alpha x \mid \alpha \geqslant 0\}$ of $\mathcal{K}$, such that if $x=\alpha y+(1-\alpha) z$, for $0<\alpha<1$, and $y, z \in \mathcal{K}$, one can conclude that $y, z \in E[8,9]$. The dual cone of a set $S \subseteq \mathcal{H}$, denoted by $S^{*}$, is defined to be

$$
S^{*}=\{y \in \mathcal{H} \mid\langle x, y\rangle \geqslant 0, \forall x \in S\} .
$$

If $S$ is a pointed closed convex cone, then the interior of its dual cone, int $S^{*}$, is given by

$$
\text { int } S^{*}=\{y \in \mathcal{H} \mid\langle x, y\rangle>0, \forall x \in S, x \neq 0\}
$$

A closed convex cone in a finite dimensional Hilbert space is pointed if and only if its dual is solid [14]. It can easily be shown that $S^{*}$ is always a convex set, and that if $S_{1} \subseteq S_{2}$, then $S_{2}^{*} \subseteq S_{1}^{*}$. In addition, $S=\left(S^{*}\right)^{*}$, if and only if $S$ is a closed convex cone. For more on convexity, cones, and their duals the reader is referred to Berman [2], Rockafellar [18], and Stoer and Witzgall [21].

In Sections 3.3 and 3.5, we shall be referring to two properties of a linear map that we now define. Given a pointed closed convex cone $\mathcal{K} \subseteq \mathcal{H}$, a linear map $M: \mathcal{H} \rightarrow \mathcal{H}$ is called $\mathcal{K}$-positive if for all $0 \neq X \in \mathcal{K}, M(X) \in$ int $\mathcal{K}^{*}$. Furthermore, a linear map $M: \mathcal{H} \rightarrow \mathcal{H}$ is called $\mathcal{K}$-copositive if $\langle X, M(X)\rangle \geqslant 0$, for all $X \in \mathcal{K}[2,6,11]$.

We are now ready to formulate the cone problems that are considered in the paper. The Cone-LP is formulated as follows: Given a cone $\mathcal{K} \subseteq \mathcal{H}$, a linear map $M: \mathcal{H} \rightarrow \mathcal{H}$, and the elements $Q$ and $C$ in $\mathcal{H}$, find $Z \in \mathcal{H}$ (if it exists) as a solution to:

$$
\begin{aligned}
& \min \langle C, Z\rangle, \\
& Z \in \mathcal{K}, \\
& Q+M(Z) \in \mathcal{K}^{*} .
\end{aligned}
$$

Similarly, the Cone-LCP is formulated as follows: Given a cone $\mathcal{K} \subseteq \mathcal{H}$, a linear map $M: \mathcal{H} \rightarrow \mathcal{H}$, and $Q \in \mathcal{H}$, find $Z \in \mathcal{H}$ (if it exists) such that:

$$
\begin{aligned}
& Z \in \mathcal{K}, \\
& Q+M(Z) \in \mathcal{K}^{*}, \\
& \langle Z, Q+M(Z)\rangle=0 .
\end{aligned}
$$

The above instances of the Cone-LP and the Cone-LCP shall be referred to as the

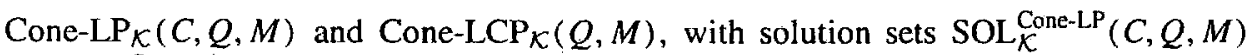
and $\mathrm{SOL}_{\mathcal{K}}^{\text {Cone-LCP }}(Q, M)$, respectively. When $\mathcal{K}$ is the nonnegative orthant in the $n$-dimensional Euclidean space, the Cone-LP $\mathcal{K}_{\mathcal{C}}(C, Q, M)(7)-(9)$ and the Cone-LCP $\mathcal{K}(Q, M)$ (10)-(12), are equivalent to the familiar LP and the LCP. We shall also find it convenient to refer to the problem of finding a feasible element in the Cone-LP, i.e., an 
element that satisfies $(8)-(9)$, as a Cone-LP $\mathrm{C}_{\mathcal{K}}(*, Q, M)$. In this case the solution set is denoted by $\operatorname{SOL}_{\mathcal{K}}^{\text {Cone-LP }}(*, Q, M)$.

A problem which serves as a bridge between the BMI and the Cone-LP/LCPs is what we have referred to as the Extreme Form Problem (EFP): Given a pointed closed convex cone $\mathcal{K} \subseteq \mathcal{H}$, a linear map $M: \mathcal{H} \rightarrow \mathcal{H}$, find $X \in \mathcal{H}$ (if it exists), such that,

$$
\begin{aligned}
& X \in \mathcal{K}, \\
& M(X) \in \operatorname{int} \mathcal{K}^{*}, \\
& X \text { is an extreme form of } \mathcal{K},
\end{aligned}
$$

where the "int $\mathcal{K}$ " denotes the interior of the cone $\mathcal{K}$. The above instance of the EFP is referred to as the $\operatorname{EFP}_{\mathcal{K}}(M)$, with the solution set $\operatorname{SOL}_{\mathcal{K}}^{\mathrm{EFP}}(M)$. As we mentioned in the Introduction, when $\mathcal{K}$ is the nonnegative orthant in the $n$-dimensional Euclidean space, the EFP is a trivial problem. It should be noted that $\operatorname{SOL}_{\mathcal{K}}^{\operatorname{EFP}}(M)$ is nonconvex; given the two extreme forms of $\mathcal{K}$ that solve the $\operatorname{EFP}_{\mathcal{K}}(M)$, a strict convex combination of them is not an extreme form of $\mathcal{K}$. It is also important to note that the EFP requires $M(X)$ to lie in the interior of the dual cone. This is in light of the fact that for certain important classes of linear maps $M$, including the map that is encountered in the context of the BMIs, $M(X)$ is known to lie in, but possibly on the boundary of, the dual cone, for all the extreme forms $X$ of the cone $\mathcal{K}$. It is still not clear how to adapt the interior point methods for the Semi-Definite Programming [1,17], for solving the EFP (for example, over the cone of positive semi-definite matrices). This is the main reason why we have chosen to relate the EFP that arises from the BMIs, to a Cone-LP, and subsequently to a Cone-LCP over the cone of positive semi-definite matrices.

The Cone-LP/LCP formulation of the BMI rests upon the introduction of two classes of matrices. These matrix classes are a generalization of the cone of completely positive matrices $(B)$, and its dual, the cone of copositive matrices $(C)$. Both $B$ and $C$ are closely related to another matrix cone, the positive semi-definite matrices (PSD). The PSD has received much attention recently in the context of SDPs, the linear complementarity problems over matrix cones, and the behavior of the interior point methods for solving them $[1,13,17]$. The cones $B$ and $C$ have been studied in various settings. The matrix cone $B$, which is the set of matrices with quadratic forms expressible as a sum of squares of linear forms, has been studied in the context of block design in combinatorial theory [8]. The matrix cone $C$, which is the set of matrices with quadratic forms nonnegative over the nonnegative orthant, has been studied in the context of the LCP [4].

The interior point methods for SDPs have not been adapted for the matrix cones $B$ and $C$ (and their generalizations which are introduced in this paper). This is due to the fact that even checking whether a given matrix is copositive is a difficult computational task [16]. However, as it is shown in Section 3.5, the Cone-LCP that arises from the BMI can be formulated over the cone of positive semi-definite matrices, and consequently, it is readily amenable to an interior point approach $[1,17]$. Moreover, it has been recently shown that the monotone Cone-LCP over the PSD cone can in fact be reduced to 
an SDP, and therefore can be solved using the interior point algorithms for the SDP directly [12].

\subsection{Notation}

$A^{\prime} \quad$ Transpose of the matrix $A$.

$A_{i j} \quad$ The element of matrix $A$ located in the $i$ th row and $j$ th column.

$\operatorname{diag}(y)$ The (square) diagonal matrix of the appropriate dimension, with vector $y$ on its diagonal.

$x>y \quad$ The strict partial ordering: $x_{i}>y_{i}, i=1, \ldots, n$.

$x \geqslant y \quad$ The partial ordering: $x_{i} \geqslant y_{i}, i=1, \ldots, n$.

$I_{p \times p} \quad$ The $p \times p$ identity matrix.

$\mathbb{R}^{p} \quad$ The $p$-dimensional Euclidean space.

$\mathbb{R}_{+}^{p} \quad$ The non-negative orthant in the $p$-dimensional Euclidean Space.

$\mathbb{R}^{p \times p} \quad$ The space of $p \times p$ matrices with real entries.

$\mathrm{SR}^{p \times p} \quad$ Symmetric $p \times p$ matrices with real entries.

$\widehat{\mathrm{S}} \mathbb{R}^{p \times p} \quad$ Skew-symmetric $p \times p$ matrices with real entries.

$\mathrm{SR}_{+}^{p \times p} \quad$ Symmetric $p \times p$ positive semi-definite matrices with real entries.

$\mathrm{SR}_{++}^{p \times p} \quad$ Symmetric $p \times p$ positive definite matrices with real entries.

$A \succ B \quad A-B$ is symmetric positive definite.

$A \succeq B \quad A-B$ is symmetric positive semi-definite.

$A \bullet B \quad$ Trace $A B^{\prime}$, the inner product used for the (Hilbert) space of matrices.

$A \otimes B \quad$ The Kronecker product of matrices $A$ and $B$.

$\operatorname{vec} M \quad$ The vector obtained by stacking up the columns of the matrix $M$.

$C^{*} \quad$ The dual of the set $C$.

int $A$ The interior of the set $A$.

$\dot{\mathcal{B}} \quad$ The set of extreme forms (rays) of the cone $\mathcal{B}$.

\section{The Cone-LP and the Cone-LCP formulation of the BMI}

In this section we discuss the formulation of the BMI feasibility problem as a Cone-LP over a suitable generalization of the cone of completely positive matrices and subsequently, as a Cone-LCP over the cone of positive semi-definite matrices. This is done by first reducing the BMI to an EFP, and subsequently reducing the EFP to a ConeLP/LCP. As it becomes evident, various embeddings of matrices in different dimensions are needed to make these reductions as transparent as possible. For this purpose the vec notation, which is used in the studying of Kronecker products, has become specially handy. The vec operator, applied to a matrix in $\mathbb{R}^{p \times p}$, simply stacks up the columns of the matrix from left to right, and forms a vector in $\mathbb{R}^{p^{2}}[9,10]$. 


\subsection{Few initial steps}

Consider again the BMI feasibility problem: Given $H_{i j}=H_{i j}^{\prime} \in \mathrm{SR}^{p \times p}$ (the symmetric $p \times p$ matrices with real entries), does there exist $x_{i}$ 's $(1 \leqslant i \leqslant n)$, and $y_{j}$ 's $(1 \leqslant j \leqslant$ $m)$, such that

$$
\sum_{i} \sum_{j} x_{i} y_{j} H_{i j} \succ 0
$$

Let us rewrite (16) as:

$$
\sum_{i} x_{i} \sum_{j} y_{j} H_{i j}=\sum_{i} x_{i} H_{i}^{r} \succ 0
$$

where

$$
H_{i}^{y}=\sum_{j} y_{j} H_{i j} \in \mathrm{SR}^{p \times p} .
$$

As it becomes apparent by the subsequent developments, it is convenient to assume that $m=p$ and that $y_{j}$ 's $(1 \leqslant j \leqslant m)$, are nonnegative. The first assumption is made to avoid defining inner products between matrix classes of different dimensions. The second assumption is made to facilitate the dual cone characterization in the EFP and Cone-LP approaches discussed in Sections 3.3 and 3.4 (we shall later drop the nonnegativity assumption on the vector $y$ in Section 3.5, where the Cone-LCP is presented). These assumptions are warranted for the following reason. First, note that if we define $H_{j}^{x}=$ $\sum_{i} x_{i} H_{i j} \in \mathrm{SR}^{p \times p}(1 \leqslant j \leqslant m)$, then $\sum_{i} x_{i} H_{i}^{\prime}=\sum_{j} y_{j} H_{j}^{x}$. But the last sum is a linear inequality in $H_{j}^{x}$ 's. Thus, as it is customary in the Linear Programming, one can assume that $m \leqslant p$ and that $y_{j}$ 's are positive (by an appropriate augmentation). Now we would only need to define $H_{i j} \equiv 0(1 \leqslant i \leqslant n, m<j \leqslant p)$, for the assumption $m=p$ to be justified.

Remark 1. Henceforth, we shall reserve the notations " $\succeq$ " and " $\succ$ " to indicate positive semi-definiteness and positive definiteness for the $p \times p$ matrices, respectively.

Recall that Gordan's theorem of alternative $[4,21]$, relates the solvability of the following two systems of linear inequalities: Given $A \in \mathbb{R}^{n \times n}$, the system $A x>0$ has a solution if and only if the system $y^{\prime} A=0, y \geqslant 0, y \neq 0$, has no solution. This theorem can be generalized for the linear inequalities over matrix cones as follows:

Proposition 2. Given the symmetric matrices $A_{i}^{\prime} s \in \mathrm{SR}^{p \times p}(1 \leqslant i \leqslant n)$, the system $\sum_{i=1}^{n} x_{i} A_{i} \succ 0$, has a solution if and only if the system

$$
A_{i} \bullet Z=0 \quad(1 \leqslant i \leqslant n), \quad Z \succeq 0, \quad Z \neq 0
$$

has no solution. 
Many variants of Proposition 2 and the generalizations of the other theorems of alternatives for a finite dimensional cone, have been discussed by Berman [2], and we shall therefore omit the proof of this result.

From Gordan's theorem of alternative over the cone of symmetric positive semidefinite matrices, one concludes that the BMI (16) does not have a solution if and only if,

$$
(\forall y \geqslant 0)(\exists Z \succeq 0, Z \neq 0): \quad H_{i}^{y} \bullet Z=0 .
$$

Therefore, the BMI (16) has a solution if and only if,

$$
(\exists y \geqslant 0)(\forall Z \succeq 0, Z \neq 0): \quad \sum_{i}\left(H_{i}^{y} \bullet Z\right)^{2}>0 .
$$

Remark 3. We note that if (19) is satisfied, and that the variable $y$ is found by a certain procedure, then the BMI (16) is reduced to a Linear Matrix Inequality (LMI), which can be solved efficiently by the interior point methods for the variable $x$.

The statement of Remark 3 follows from the following observation: Suppose $y$ is found such that for all $Z \succeq 0, Z \neq 0, \sum_{i}\left(H_{i}^{\prime \prime} \bullet Z\right)^{2}>0$. Therefore, according to Proposition 2,

$$
Z \succeq 0, \quad Z \neq 0, \quad H_{i}^{\prime} \bullet Z=0(1 \leqslant i \leqslant n),
$$

has no solution. Therefore $\sum_{i=1}^{n} x_{i} H_{i}^{y} \succ 0$ has a solution. The last inequality is indeed an LMI.

Now let,

$$
H_{i}=[\overbrace{\operatorname{vec} H_{i 1} 0 \ldots 0}^{p}, \overbrace{0 \operatorname{vec} H_{i 2} \ldots 0}^{p}, \ldots, \overbrace{00 \ldots \operatorname{vec} H_{i p}}^{p}] \in \mathbb{R}^{p^{2} \times p^{2}}
$$

and $Y=\operatorname{diag}(y) \in \mathrm{SR}^{p \times p}$. Since $\left(H_{i}^{\prime}\right)^{\prime}=H_{i}^{\prime}=\sum_{j} y_{j} H_{i j}$ (recalling that $H_{i j}$ 's are symmetric matrices),

$$
\operatorname{vec}\left(H_{i}^{\prime \prime}\right)^{\prime}=H_{i} \operatorname{vec} Y
$$

thereby,

$$
H_{i}^{y} \cdot Z=\left(\operatorname{vec}\left(H_{i}^{y}\right)^{\prime}\right)^{\prime}(\operatorname{vec} Z)=\left(H_{i} \operatorname{vec} Y\right)^{\prime}(\operatorname{vec} Z)=(\operatorname{vec} Y)^{\prime} H_{i}^{\prime}(\operatorname{vec} Z) .
$$

Combining (19) and (22) we conclude that (16) has a solution if and only if there exists $Y \succeq 0, Y=\operatorname{diag}(y)$, for some $y \geqslant 0$, such that for all $Z \succeq 0, Z \neq 0$,

$$
(\operatorname{vec} Z)^{\prime}\left\{\sum_{i} H_{i}(\operatorname{vec} Y)(\operatorname{vec} Y)^{\prime} H_{i}^{\prime}\right\}(\operatorname{vec} Z)>0 .
$$

Let $X=(\operatorname{vec} Y)(\operatorname{vec} Y)^{\prime}, Y \in \mathbb{R}^{p \times p}$, and,

$$
M(X)=\sum_{i} H_{i} X H_{i}^{\prime} \text {. }
$$


Two key observations, due to the particular form of the linear map $M$ (24), are in order at this point:

(1) According to (20), for all $p \times p$ skew-symmetric matrices $Z, H_{i}$ vec $Z=0$ $(i=1, \ldots, n)$. Consequently, if there exists a matrix $Y \in \mathbb{R}^{p \times p}$ such that (23) bolds, then one can assume that $Y$ is symmetric, since the skew-symmetric part of $Y$ does not contribute to the left-hand side of the inequality (23): Let $Y=Y_{1}+Y_{2}$, with $Y_{1}$ and $Y_{2}$ being the symmetric and skew-symmetric part of $Y$, respectively. Then for $1 \leqslant i \leqslant n, H_{i} \operatorname{vec} Y=H_{i}\left(\operatorname{vec} Y_{1}+\operatorname{vec} Y_{2}\right)=H_{i} \operatorname{vec} Y_{1}$.

(2) According to (21), for all matrices $Y \in \mathbb{R}^{p \times p}, H_{i} \operatorname{vec} Y=\operatorname{vec} W_{i}$, for some $W_{i} \in S \mathbb{R}^{n \times p}$. Therefore if $X=(\operatorname{vec} Y)(\operatorname{vec} Y)^{\prime}$, then $M(X)$ can be represented by

$$
M(X)=\sum_{i}\left(\operatorname{vec} W_{i}\right)\left(\operatorname{vec} W_{i}\right)^{\prime} .
$$

We shall use these observations in Section 3.5.

Remark 4. Suppose that the vector $y$ is not required to be nonnegative in the above analysis. It is clear that the above steps are still valid with the obvious modifications, and that the end result would read as follows: The BMI has a solution if and only if there exists a diagonal matrix $Y$, such that for all $Z \succeq 0, Z \neq 0$, the inequality (23) holds. We shall use this observation later in Section 3.5.

The inequality (23) can be interpreted as requiring $M(X)$ to belong to a certain matrix class. The matrices in this class are symmetric (given that $X$ is symmetric) and have quadratic forms which are positive over the vec form of the non-zero matrices in $\mathrm{SR}_{+}^{\prime \times p}$. This observation justifies the introduction of certain matrix classes which we shall discuss next.

\subsection{Few matrix cones}

At this point let us introduce certain classes of matrices. We then delineate (23) in terms of these matrix classes, which in turn facilitate the transition from the BMI to the EFP. In what follows it is assumed that all the matrix classes are subsets of $S \mathbb{R}^{p^{2} \times p^{2}}$, and that the duals of matrix classes are taken with respect to this linear space.

Denote by $\mathcal{P S D}$, the class of $p^{2} \times p^{2}$ symmetric positive semi-definite matrices, i.e., matrices for the which the quadratic form is nonnegative over the vec form of the $p \times p$ matrices $\left(\mathbb{R}^{p \times p}\right)$,

$$
\mathcal{P S D}=\left\{A \in \mathrm{SR}^{\prime \nu^{2} \times p^{2}} \mid(\operatorname{vec} Z)^{\prime} A(\operatorname{vec} Z) \geqslant 0, Z \in \mathbb{R}^{p \times p}\right\} .
$$

Let $\mathcal{P S D _ { 0 }}$ denote the subset of $p^{2} \times p^{2}$ symmetric matrices with quadratic forms nonnegative over the vec form of the symmetric $p \times p$ matrices $\left(\mathrm{SR}^{p \times p}\right)$, and with the vec form of the skew-symmetric matrices $\left(\widehat{\mathrm{S}} \mathbb{R}^{p \times p}\right)$ in their null space, i.e., 


$$
\begin{aligned}
& \mathcal{P} \mathcal{S D}_{0}=\left\{A \in \mathrm{SR}^{p^{2} \times p^{2}} \mid(\operatorname{vec} Z)^{\prime} A(\operatorname{vec} Z) \geqslant 0, Z \in \mathbb{S R}^{p \times p},\right. \\
& \left.A(\operatorname{vec} W)=0 \text {, for all } W \in \widehat{\mathrm{S}} \mathbb{R}^{p \times p}\right\} \text {. }
\end{aligned}
$$

Clearly both $\mathcal{P S D}(26)$ and $\mathcal{P S} \mathcal{D}_{0}(27)$ are closed convex cones. It is well known that the matrix class $\mathcal{P S D}$ has the following properties:

(1) For all rank $t \geqslant 1$ matrices $A \in \mathcal{P S D}$, there are non-zero matrices $W_{i} \in \mathbb{R}^{p \times p}$ $(1 \leqslant i \leqslant t)$, such that,

$$
A=\sum_{i=1}^{\prime}\left(\operatorname{vec} W_{i}\right)\left(\operatorname{vec} W_{i}\right)^{\prime}
$$

and $W_{i} \bullet W_{j}=0(i \neq j)[9]$.

(2) $\mathcal{P S D}=\mathcal{P S D}^{*}$. Moreover, $\mathcal{P S D}$ is pointed and solid [2].

(3) The extreme forms of $\mathcal{P S D}$ are of the form $(\operatorname{vec} W)(\operatorname{vec} W)^{\prime}, W \in \mathbb{R}^{p \times p}$ [9]. Before we state the next result, we make a note of the following fact.

Proposition 5. For all $Z \in \mathrm{SR}^{p \times p}$ and $W \in \widehat{\mathrm{S}} \mathbb{R}^{p \times p},(\operatorname{vec} Z)^{\prime}(\operatorname{vec} W)=Z \bullet W=0$.

Proof. Let $W=A-A^{\prime}$ for some $A \in \mathbb{R}^{p \times p}$. Then

$$
Z \bullet W=Z \bullet A-Z \bullet A^{\prime}=0 .
$$

The following proposition states that certain essential features of the $\mathcal{P S D}$ cone can be generalized for the class of $\mathcal{P} S \mathcal{D}_{0}$ matrices.

Proposition 6. The matrix class $\mathcal{P} \mathcal{S D}_{0}$ has the following properties:

(1) For all rank $t \geqslant 1$ matrices $A \in \mathcal{P} S \mathcal{D}_{0}$, there are non-zero symmetric matrices $W_{i} \in \mathbb{S}^{p \times p}(1 \leqslant i \leqslant t)$, such that,

$$
A=\sum_{i=1}^{\prime}\left(\operatorname{vec} W_{i}\right)\left(\operatorname{vec} W_{i}\right)^{\prime}
$$

and $W_{i} \bullet W_{j}=0(i \neq j)$. Furthermore, any matrix which has such a representation is in $\mathcal{P S D _ { 0 }}$.

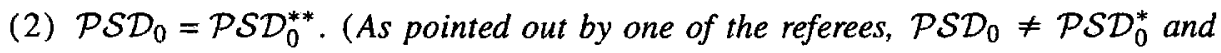
$\mathcal{P S D _ { 0 }}$ is not solid.)

(3) The extreme forms of $\mathcal{P S D _ { 0 }}$ are of the form $(\operatorname{vec} W)(\operatorname{vec} W)^{\prime}, W \in \mathrm{SR}^{p \times p}$.

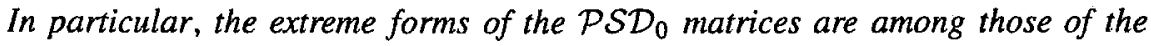
$\mathcal{P S D}$ cone.

Proof. (1) We first establish the second statement of (1). For all matrices $Z \in \mathrm{SR}^{p \times p}$, $(\operatorname{vec} Z)^{\prime} A(\operatorname{vec} Z)=\sum_{i}\left(Z \bullet W_{i}\right)^{2} \geqslant 0$ 
$A(\operatorname{vec} W)=\sum_{i}\left(\operatorname{vec} W_{i}\right)\left(\operatorname{vec} W_{i}\right)^{\prime}(\operatorname{vec} W)=0$

for all $W \in \widehat{\mathrm{S}} \mathbb{R}^{p \times p}$, by Proposition 5. Therefore $A \in \mathcal{P} \mathcal{S D _ { 0 }}$.

Define

$$
P:=\left\{A \mid A=\sum_{i}\left(\operatorname{vec} W_{i}\right)\left(\operatorname{vec} W_{i}\right)^{\prime}, W \in \mathrm{SR}^{p \times p}\right\}
$$

It will be shown that in fact $P=\mathcal{P} S \mathcal{D}_{0}$.

Let $A=\sum_{i}\left(\operatorname{vec} W_{i}\right)\left(\operatorname{vec} W_{i}\right)^{\prime} \in P$, for some symmetric matrices $W_{i}$ 's. Then for all $Z \in \mathrm{SR}^{p \times p}$,

$$
\begin{aligned}
(\operatorname{vec} Z)^{\prime} A(\operatorname{vec} Z) & =(\operatorname{vec} Z)^{\prime} \sum_{i}\left(\operatorname{vec} W_{i}\right)\left(\operatorname{vec} W_{i}\right)^{\prime}(\operatorname{vec} Z) \\
& =\sum_{i}\left(Z \bullet W_{i}\right)^{2} \geqslant 0 .
\end{aligned}
$$

Moreover, for all $Z \in \widehat{S} \mathbb{R}^{p \times p}$,

$$
A(\operatorname{vec} Z)=\left(\sum_{i}\left(\operatorname{vec} W_{i}\right)\left(\operatorname{vec} W_{i}\right)^{\prime}\right)(\operatorname{vec} Z)=0
$$

as a result of Proposition 5. Therefore $P \subseteq \mathcal{P S D _ { 0 }}$.

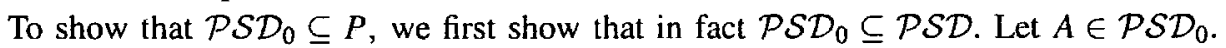
Since for all $W \in \mathbb{R}^{p \times p}, W$ can be written in the form $W=W_{1}+W_{2}$, with $W_{1} \in \mathrm{SR}^{p \times p}$ and $W_{2} \in \widehat{S}^{p \times p}$, one has

$$
\begin{aligned}
(\operatorname{vec} W)^{\prime} A(\operatorname{vec} W) & =\left(\operatorname{vec} W_{1}+\operatorname{vec} W_{2}\right)^{\prime} A\left(\operatorname{vec} W_{1}+\operatorname{vec} W_{2}\right) \\
& =\left(\operatorname{vec} W_{1}\right)^{\prime} A\left(\operatorname{vec} W_{1}\right) \geqslant 0
\end{aligned}
$$

since $A\left(\operatorname{vec} W_{2}\right)=0$. Therefore $A \in \mathcal{P S D}$. Suppose that $A$ has rank $t \geqslant 1$. Then there exist non-zero matrices $U_{i} \in \mathbb{R}^{p \times p}(i=1, \ldots, t)$, such that,

$$
A=\sum_{i=1}^{\prime}\left(\operatorname{vec} U_{i}\right)\left(\operatorname{vec} U_{i}\right)^{\prime}
$$

and $\left(\operatorname{vec} U_{i}\right)^{\prime}\left(\operatorname{vec} U_{j}\right)=U_{i}^{\prime} \bullet U_{j}=0(i \neq j)$. It now suffices to show that all matrices $U_{i}$ 's in (30) should be symmetric. For this purpose, we will perform an induction on $t \geqslant 1$.

Let $A=(\operatorname{vec} U)(\operatorname{vec} U)^{\prime}, 0 \neq U \in \mathbb{R}^{p \times p}, U=\bar{U}+\widetilde{U}$, with $\bar{U} \in \mathrm{S}^{p \times p}$ and $\tilde{U} \in \widehat{\mathrm{S}} \mathbb{R}^{p \times p}$. Then,

$$
A(\operatorname{vec} \widetilde{U})=(\operatorname{vec} U)(\operatorname{vec} U)^{\prime}(\operatorname{vec} \widetilde{U})=\|\widetilde{U}\|^{2}(\operatorname{vec} U) .
$$

Since $A(\operatorname{vec} \widetilde{U})=0$ by the definition of $\mathcal{P S D _ { 0 }}$, we conclude that $\widetilde{U}=0$. 
For $t>1$, suppose that the proposition holds for $t-1$, that is,

$$
A=\sum_{i=1}^{t-1}\left(\operatorname{vec} U_{i}\right)\left(\operatorname{vec} U_{i}\right)^{\prime}+(\operatorname{vec} W)(\operatorname{vec} W)^{\prime},
$$

with $U_{i} \in S \mathbb{R}^{p \times p},(1 \leqslant i \leqslant t-1)$, and $W \in \mathbb{R}^{p \times p}$; by (27) and Proposition 5 , this also establishes that $\sum_{i=1}^{t-1}\left(\operatorname{vec} U_{i}\right)\left(\operatorname{vec} U_{i}\right)^{\prime} \in \mathcal{P} \mathcal{S} \mathcal{D}_{0}$. We like to show that in fact $W \in S \mathbb{R}^{p \times p}$.

Proceeding in exactly the same way as we did for the case of $t=1$, we form $A(\operatorname{vec} \widetilde{W})$, where $W=\bar{W}+\widetilde{W}, \bar{W} \in \mathrm{S}^{p \times p}$, and $\tilde{W} \in \widehat{S} \mathbb{R}^{p \times p}$. Now since $\left(\operatorname{vec} U_{i}\right)^{\prime}(\operatorname{vec} \tilde{W})=0$ $(1 \leqslant i \leqslant t-1)$, by Proposition 5 , we again conclude that $\|\widetilde{W}\|^{2}(\operatorname{vec} W)=0$. Therefore $\widetilde{W}=0$, and $W \in \mathrm{SR}^{p \times p}$.

Hence, all matrices $U_{i}$ 's in (30) are symmetric.

(2) As pointed out previously, $\mathcal{P} S \mathcal{D}_{0}$ is a closed convex cone. Therefore $\mathcal{P} \mathcal{S D}_{0}=$

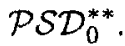

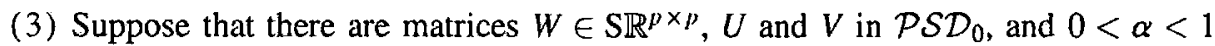
such that $(\operatorname{vec} W)(\operatorname{vec} W)^{\prime}=\alpha U+(1-\alpha) V$. It suffices to show that both $U$ and $V$ are constant multiple of $(\mathrm{vec} W)(\operatorname{vec} W)^{\prime}$.

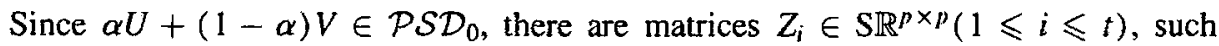
that $(\operatorname{vec} W)(\operatorname{vec} W)^{\prime}=\sum_{i=1}^{t}\left(\operatorname{vec} Z_{i}\right)\left(\operatorname{vec} Z_{i}\right)^{\prime}$ (by Part 1 of the proposition). If for

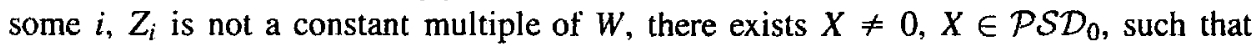
$(\operatorname{vec} W)(\operatorname{vec} W)^{\prime} \bullet X=0$, and $\left(\operatorname{vec} Z_{i}\right)\left(\operatorname{vec} Z_{i}\right)^{\prime} \bullet X>0$ (there exists $U \in S \mathbb{R}^{p \times p}$ such that $U \bullet W=0$, but $U \bullet Z_{i} \neq 0$; let $\left.X=(\operatorname{vec} U)(\operatorname{vec} U)^{\prime} \in \mathcal{P} \mathcal{S} \mathcal{D}_{0}\right)$. But this consequence contradicts our assumption that $(\operatorname{vec} W)(\operatorname{vec} W)^{\prime}=\sum_{i=1}^{t}\left(\operatorname{vec} Z_{i}\right)\left(\operatorname{vec} Z_{i}\right)^{\prime}$.

Let $\mathcal{C}$ denote the class of symmetric PSD-copositive matrices,

$$
\mathcal{C}=\left\{A \in \mathrm{SR}^{p^{2} \times p^{2}} \mid(\operatorname{vec} Z)^{\prime} A(\operatorname{vec} Z) \geqslant 0, Z \succeq 0\right\}
$$

A particular subset of $\mathcal{C}$ which be useful in our later cone formulations is the class of matrices in $\mathcal{C}$ which have the $p \times p$ skew-symmetric matrices in their null space, i.e.,

$$
\mathcal{C}_{0}:=\left\{A \in \mathcal{C} \mid A \operatorname{vec} W=0, W \in \widehat{S} \mathbb{R}^{p \times p}\right\}
$$

Finally, let $\mathcal{B}$ denote the class of symmetric PSD-completely positive matrices,

$$
\mathcal{B}=\left\{A \in \mathbb{S R}^{p^{2} \times p^{2}} \mid A=\sum_{i=1}^{t}\left(\operatorname{vec} Z_{i}\right)\left(\operatorname{vec} Z_{i}\right)^{\prime}, Z_{i} \succeq 0, t \geqslant 1\right\} .
$$

Remark 7. The matrix classes $\mathcal{C}$ and $\mathcal{B}$ are generalizations of the symmetric copositive and the completely positive matrices, respectively. Note that the matrices in class $\mathcal{C}$ have quadratic forms which are nonnegative over the vec form of the symmetric positive semi-definite matrices. 
It should be noted that many results in the context of copositive matrices [4] can also be proven for the PSD-copositive matrices. We also observe that $\mathcal{B} \subseteq \mathcal{P S D _ { 0 }} \subseteq \mathcal{P S D} \subseteq$

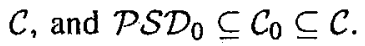

It is now observed that Eq. (23) states whether a nonlinear combination of matrices $H_{i}^{\prime} s \in \mathbb{R}^{r^{2} \times p^{2}} \quad(1 \leqslant i \leqslant n)$, belongs to the interior of the cone of PSD-copositive matrices $\mathcal{C}$. In fact, due the particular form of the linear map $M(25), M(X)$ is required to be in $\mathcal{C}_{1}:=\mathcal{C}_{0} \cap$ int $\mathcal{C}$.

We now establish that the matrix classes $\mathcal{B}$ and $\mathcal{C}$ are in fact closed convex cones. In addition, we show that they are the dual of each other, $\mathcal{C}$ is solid, and $\mathcal{B}$ is pointed. These results will facilitate the reduction of the BMI to an EFP, and subsequently to a Cone-LP/LCP. These proofs are not significantly different from those provided by $M$. Hall for the classes of completely positive and copositive matrices [8].

Lemma 8. The matrix classes $\mathcal{B}, \mathcal{C}$, and $\mathcal{C}_{0}$ are closed convex cones in $\mathrm{SR}_{\mathbb{R}^{p^{2}} \times p^{2}}$. Moreover, $\mathcal{B}^{*}=\mathcal{C}, \mathcal{C}^{*}=\mathcal{B}, \mathcal{C}$ is solid, and $\mathcal{B}$ is pointed.

Proof. $\mathcal{C}$ is a cone since if $(\operatorname{vec} Z)^{\prime} A(\operatorname{vec} Z) \geqslant 0$, then for all $\alpha \geqslant 0$,

$(\operatorname{vec} Z)^{\prime}(\alpha A)(\operatorname{vec} Z)=\alpha(\operatorname{vec} Z)^{\prime} A(\operatorname{vec} Z) \geqslant 0$.

Therefore $\alpha \mathcal{C} \subseteq \mathcal{C}$. Moreover, if $A, B \in \mathcal{C}$, then

$$
(\operatorname{vec} Z)^{\prime}(A+B)(\operatorname{vec} Z)=(\operatorname{vec} Z)^{\prime} A(\operatorname{vec} Z)+(\operatorname{vec} Z)^{\prime} B(\operatorname{vec} Z) \geqslant 0 .
$$

Therefore $\mathcal{C}+\mathcal{C} \subseteq \mathcal{C}$ and $\mathcal{C}$ is a convex cone. From the definition of $\mathcal{C}(31)$ it is clear that $\mathcal{C}$ is closed. Similarly the class $\mathcal{C}_{0}$ can be shown to be a convex cone. Moreover, if $\left\{A_{k}\right\}$ is a sequence of matrices in $\mathcal{C}_{0}$ and $A_{k} \rightarrow A$, then for all $W \in \widehat{\mathrm{S}} \mathbb{R}^{p \times p}$,

$$
\left\|A(\operatorname{vec} W)-A_{k}(\operatorname{vec} W)\right\| \leqslant\left\|A-A_{k}\right\|\|\operatorname{vec} W\|
$$

and therefore $A \in \mathcal{C}_{0}$.

$\mathcal{B}$ is a convex cone since if $A=\sum_{i=1}^{t}\left(\operatorname{vec} Z_{i}\right)\left(\operatorname{vec} Z_{i}\right)^{\prime}$, for some $t \geqslant 1$, then for all $\alpha \geqslant 0, \alpha A=\sum_{i=1}^{\prime}\left(\beta \operatorname{vec} Z_{i}\right)\left(\beta \operatorname{vec} Z_{i}\right)^{\prime}$, where $\beta=\sqrt{\alpha}$, i.e., $\alpha \mathcal{B} \subseteq \mathcal{B}$. Also from the definition of $\mathcal{B}$ it is clear that, for all $A, B \in \mathcal{B}, A+B \in \mathcal{B}$. Therefore $\mathcal{B}$ is a convex cone.

It is now shown that $\mathcal{B}^{*}=\mathcal{C}$. To show that $\mathcal{B}^{*} \subseteq \mathcal{C}$ we note that if $A \in \mathcal{B}^{*}$, then for all $Z \succeq 0$,

$$
A \bullet(\operatorname{vec} Z)(\operatorname{vec} Z)^{\prime}=(\operatorname{vec} Z)^{\prime} A(\operatorname{vec} Z) \geqslant 0
$$

and therefore $A \in \mathcal{C}$. For the relation $\mathcal{C} \subseteq \mathcal{B}^{*}$, let $A \in \mathcal{C}$. Then,

$$
A \cdot(\operatorname{vec} Z)(\operatorname{vec} Z)^{\prime} \geqslant 0 \quad(\forall Z \succeq 0)
$$

and thereby $A \bullet E \geqslant 0$, for all $E \in \mathcal{B}$. Hence $A \in \mathcal{B}^{*}$ and consequently, $\mathcal{C}=\mathcal{B}^{*}$.

We now proceed to demonstrate that $\mathcal{B}$ is closed. Granted that this is proved, it would then follow that $\mathcal{B}=\left(\mathcal{B}^{*}\right)^{*}=\mathcal{C}^{*}$. 
From Carathéodory's Theorem [18], it is known that all matrices in $\mathcal{B}$ can be expressed as a convex combination of $N:=1+p^{2}\left(p^{2}+1\right) / 2$ matrices of the form $(\operatorname{vec} Z)(\operatorname{vec} Z)^{\prime}, Z \succeq 0$ (not necessarily distinct), where $p^{2}\left(p^{2}+1\right) / 2$ is the dimension of $\mathrm{SR}^{p^{2} \times p^{2}}$.

Let $A$ be the limit of the sequence $\left\{A_{k}\right\}_{k \geqslant 0}, A_{k} \in \mathcal{B}$, that is, there are matrices $Z_{i}^{k} \succeq 0$ such that

$$
\left\|\sum_{i=1}^{N}\left(\operatorname{vec} Z_{i}^{k}\right)\left(\operatorname{vec} Z_{i}^{k}\right)^{\prime}-A\right\| \rightarrow 0, \quad \text { as } k \rightarrow \infty .
$$

Since $N$ is fixed, the coefficients of the matrices $Z_{i}^{k}$ should stay bounded and consequently, there is a subsequence of these matrices that converges to a matrix $Z_{i}^{*} \succeq 0$ (since the cone of positive semi-definite matrices is closed). Therefore

$$
A=\sum_{i=1}^{N}\left(\operatorname{vec} Z_{i}^{*}\right)\left(\operatorname{vec} Z_{i}^{*}\right)^{\prime}
$$

Hence $A \in \mathcal{B}$, and $\mathcal{B}$ is closed.

To show that $\mathcal{C}$ has a non-empty interior, we observe that $I_{p^{2} \times p^{2}} \in \operatorname{int} \mathcal{C}$. Consequently $\mathcal{C}$ is solid, and $\mathcal{B}=\mathcal{C}^{*}$ is pointed [14].

Since we will later need an explicit expression for the extreme forms of $\mathcal{B}$, the following lemma is of importance.

Lemma 9. The extreme forms of $\mathcal{B}$ are matrices $(\operatorname{vec} Z)(\operatorname{vec} Z)^{\prime}, Z \succeq 0$.

Proof. Suppose that there are matrices $W \succeq 0, U, V \in \mathcal{B}$, and $0<\alpha<1$ such that $(\operatorname{vec} W)(\operatorname{vec} W)^{\prime}=\alpha U+(1-\alpha) V$. Our goal is to show that both $U$ and $V$ are constant multiple of $(\operatorname{vec} W)(\operatorname{vec} W)^{\prime}$. Since $\alpha U+(1-\alpha) V \in \mathcal{B}$, there are matrices $Z_{i} \succeq 0(1 \leqslant$ $i \leqslant t)$, such that $(\operatorname{vec} W)(\operatorname{vec} W)^{\prime}=\sum_{i=1}^{t}\left(\operatorname{vec} Z_{i}\right)\left(\operatorname{vec} Z_{i}\right)^{\prime}$ (by the definition of $\mathcal{B}$ ). If for some $i, Z_{i}$ is not a constant multiple of $W$, there exists $X \neq 0, X \in \mathcal{P S} \mathcal{D}_{0}$, such that $(\operatorname{vec} W)(\operatorname{vec} W)^{\prime} \bullet X=0$, and $\left(\operatorname{vec} Z_{i}\right)\left(\operatorname{vec} Z_{i}\right)^{\prime} \bullet X>0$ (there exists $U \in \mathrm{SR}^{p \times p}$ such that $U \bullet W=0$, but $U \bullet Z_{i} \neq 0$; let $\left.X=(\operatorname{vec} U)(\operatorname{vec} U)^{\prime} \in \mathcal{P} \mathcal{S D}_{0}\right)$. But this consequence contradicts our assumption that $(\operatorname{vec} W)(\operatorname{vec} W)^{\prime}=\sum_{i=1}^{l}\left(\operatorname{vec} Z_{i}\right)\left(\operatorname{vec} Z_{i}\right)^{\prime}$. Therefore any matrix of the form $(\operatorname{vec} W)(\operatorname{vec} W)^{\prime}, W \succeq 0$, cannot be written as a strict convex combination of the other elements of $\mathcal{B}$, not already on the ray $\left\{\lambda(\operatorname{vec} W)(\operatorname{vec} W)^{\prime} \mid\right.$ $\lambda \geqslant 0\}$, which is the desired result by the definition of the extreme form of a cone.

The discussion of the matrix cones $\mathcal{P S D}, \mathcal{P S} \mathcal{D}_{0}, \mathcal{B}$, and $\mathcal{C}$, which we have provided above, is sufficient for the reformulation of the BMI as an EFP, the subject which we shall examine in the following section. 


\subsection{The EFP formulation of the BMI}

We now use the results of the previous section to reformulate the BMI as the $\operatorname{EFP}_{\mathcal{B}}(M)$, where $M$ is defined by (24), and $\mathcal{B}$ is the cone of PSD-completely positive matrices.

Recall that $\operatorname{EFP}_{\mathcal{K}}(M)(13)-(15)$, formulated in a finite dimensional inner product space, is the problem of finding an extreme form of the pointed closed convex cone $\mathcal{K}$, which gets mapped by the linear map $M$, to the interior of the dual cone of $\mathcal{K}$. For a given $M$, we say that the $\operatorname{EFP}_{\mathcal{K}}(M)$ is feasible if there exists an $X$ that satisfies (13) and (14). The next result is of importance in regards to the feasibility issue. Moreover, it is the first result that connects the EFP (13)-(15) to the Cone-LCP (10)-(12).

Proposition 10. Given the linear map $M$, the problem $\operatorname{EFP}_{\mathcal{K}}(M)$ is feasible if and only if for every $Q$, the Cone-LCP $\operatorname{LC}_{\mathcal{K}}(Q, M)$ is feasible.

Proof. Suppose that the $\operatorname{EFP}_{\mathcal{K}}(M)$ is feasible. Hence there exists $X \in \mathcal{K}$, such that $M(X) \in$ int $\mathcal{K}^{*}$. Then for every $Q$ one can choose $\lambda>0$ large enough such that $Q+M(\lambda X)=Q+\lambda M(X) \in \mathcal{K}^{*}$. On the other hand, if the Cone-LCP $\mathcal{X}(Q, M)$ is feasible for every $Q$, let $Q \in \operatorname{int}\left(-\mathcal{K}^{*}\right)$, and therefore, the $\operatorname{EFP}_{\mathcal{K}}(M)$ is clearly feasible.

One can establish certain results in the context of the $\operatorname{EFP}_{\mathcal{K}}(M)$, for various classes of cones $\mathcal{K}$, and linear maps $M$, in the spirit of the complementarity problems. We will not pursue this line of investigation in the present work. Nevertheless, it should be noted that the EFP is a non-trivial problem when the cardinality of the set of extreme forms is infinite, finite but very large, or when checking whether an element is an extreme form of the cone $\mathcal{K}$ is computationally difficuit.

The importance of the $\operatorname{EFP}_{\mathcal{K}}(M)$ in the context of the BMI is established through the following result. In fact, as the next proposition states, the BMI is a special instance of the EFP.

Proposition 11. Let $\mathcal{B}$ be the class of PSD-completely positive matrices (33), and the linear map $M$ be defined by (24). Then the BMI has a solution if and only if the $\operatorname{EFP}_{\mathcal{B}}(M)$ has a solution. Moreover, the solution of one yields the solution of the other.

Proof. If the BMI has a solution $X$, then there exists $Y=\operatorname{diag}(y), y \geqslant 0, X=$ (vec $Y$ ) $(\operatorname{vec} Y)^{\prime}$, such that $M(X) \in \operatorname{int} \mathcal{C}$, and hence, the $\operatorname{EFP}_{\mathcal{B}}(M)$ has a solution.

Conversely, suppose that the $\operatorname{EFP}_{\mathcal{B}}(M)$ has a solution $X$. Then there exists $V \succeq 0$, such that $X=(\operatorname{vec} V)(\operatorname{vec} V)^{\prime}$ and $M(X) \in \operatorname{int} \mathcal{C}$, i.e., for all $Z \succeq 0, Z \neq 0$,

$$
(\operatorname{vec} Z)^{\prime}\left\{\sum_{i} H_{i}(\operatorname{vec} V)(\operatorname{vec} V)^{\prime} H_{i}^{\prime}\right\}(\operatorname{vec} Z)>0 .
$$

Let $V=T^{\prime} Y T$ be such that $Y$ is diagonal, $T$ is nonsingular, and $T^{\prime}=T^{-1}$. Then vec $Y=$ $(T \otimes T) \operatorname{vec} V$. 
We observe that

$$
\begin{aligned}
& (\operatorname{vec} Z)^{\prime}\left\{\sum_{i} H_{i}(\operatorname{vec} Y)(\operatorname{vec} Y)^{\prime} H_{i}^{\prime}\right\}(\operatorname{vec} Z) \\
& =(\operatorname{vec} Y)^{\prime}\left\{\sum_{i} H_{i}(\operatorname{vec} Z)(\operatorname{vec} Z)^{\prime} H_{i}^{\prime}\right\}(\operatorname{vec} Y) \\
& =(\operatorname{vec} V)^{\prime}(T \otimes T)^{\prime}\left\{\sum_{i} H_{i}(\operatorname{vec} Z)(\operatorname{vec} Z)^{\prime} H_{i}^{\prime}\right\}(T \otimes T)(\operatorname{vec} V) \\
& =((T \otimes T)(\operatorname{vec} Z))^{\prime}\left\{\sum_{i} H_{i}(\operatorname{vec} V)(\operatorname{vec} V)^{\prime} H_{i}^{\prime}\right\}(T \otimes T)(\operatorname{vec} Z)>0 .
\end{aligned}
$$

The last inequality follows from the fact that if vec $W=(T \otimes T) \operatorname{vec} Z, Z \succeq 0$, and $T$ is nonsingular, then $W \succeq 0$, since $W=T Z T^{\prime}$.

Therefore the diagonal matrix $Y$, is a solution to the $\operatorname{EFP}_{\mathcal{B}}(M)$. Now define the vector $y \in \mathbb{R}_{+}^{\prime \prime}$ by $y_{i}=Y_{i i}(1 \leqslant i \leqslant p)$. By Remark 3 in Section 3.1 , it is clear that one can solve for $x \in \mathbb{R}^{n}$ in the BMI problem via a semi-definite program (or an LMI).

Remark 12. Suppose that the nonnegativity assumption on the vector $y$ is dropped. Then, in view of Remark 4 and Proposition 6, it follows that the BMI is also equivalent to finding a extreme form $X$ of the $\mathcal{P S D _ { 0 }}$ cone (27) such that $M(X) \in$ int $\mathcal{B}^{*} \equiv$ int $\mathcal{C}$, and in fact, $M(X) \in \mathcal{C}_{1}:=\mathcal{C}_{0} \cap$ int $\mathcal{C}$. This observation shall be used in Section 3.5 .

The implication of Proposition 11 is that the BMI is equivalent to checking whether the image of an extreme form of the matrix cone $\mathcal{B}$ under the linear map $M$ (which is constructed from the original data of the BMI), is in the interior of the dual cone $\mathcal{B}^{*}$. This equivalence thus provides a rather simple geometric interpretation of the BMI feasibility problem.

An immediate consequence of the EFP formulation is the following characterization of the BMI instances for which a solution exists.

Proposition 13. The BMI has a solution if the linear map $M$ (24) is B-positive (see Section 2.1 for the definition of the positivity of a linear map).

Proof. If $M$ is $\mathcal{B}$-positive, then every (non-zero) extreme form of $\mathcal{B}$ is mapped to the interior of $\mathcal{B}^{*} \equiv \mathcal{C}$. Therefore the $\operatorname{EFP}_{\mathcal{B}}(M)$, and consequently the $\mathrm{BMI}$, have a solution.

In the next two sections, we shall explore the connections between the $\operatorname{EFP}_{\mathcal{B}}(M)$ and the linear programming problem defined over the matrix cone $\mathcal{B}$, and subsequently, the linear complementarity problem over the matrix cone $\mathcal{P S D}$. These approaches will suggest certain conceptual algorithms for solving the BMI. Nevertheless, the practical aspects of solving the BMI via these formulations will be elaborated on in Section 3.5 . 


\subsection{The Cone-LP formulation of the BMI}

In this section we shall explore an approach for solving the BMI based on its connection with a Cone-LP over the cone of PSD-completely positive matrices, $\mathcal{B}$. For this purpose we use the EFP formulation of the BMI, as discussed in the previous section. In particular, in the subsequent sections, we use the terms "BMI" and " $\operatorname{EFP}_{\mathcal{B}}(M)$," with the linear map $M$ defined by (24), synonymously.

Since the Cone-LP approach for the $\operatorname{EFP}_{\mathcal{B}}(M)$ is rather straightforward, we begin our discussion with the main result. Recall from Section 2.1 that $\operatorname{SOL}_{\mathcal{B}}^{\text {Cone-LP }}(*, Q, M)=$ $\left\{X \in \mathcal{B} \mid Q+M(X) \in \mathcal{B}^{*}\right\}$. We shall denote by $S(\alpha)$ the set $\operatorname{SOL}_{\mathcal{B}}^{\text {Cone-LP }}(*,-\alpha I, M)$ and by $\hat{\mathcal{B}}$ the extreme forms of the cone $\mathcal{B}$.

Theorem 14. The BMI has a solution if and only if there exists $\alpha>0$, such that $S(\alpha) \cap \dot{\mathcal{B}} \neq \emptyset$.

Proof. Let $X \in S(\alpha) \cap \dot{\mathcal{B}}$. Since $-\alpha I+M(X) \in \mathcal{B}^{*}$, it is clear that for all non-zero $Z \succeq 0$, $(\operatorname{vec} Z)^{\prime} M(X)(\operatorname{vec} Z)>0$, which implies that $M(X) \in \operatorname{int} \mathcal{B}^{*}$.

On the other hand, suppose that $X \neq 0$ is an extreme form of the cone $\mathcal{B}$ that gets mapped by $M$ to the int $\mathcal{B}^{*}$. Then for all $Z \succeq 0, Z \neq 0,\|Z\|=1,(\operatorname{vec} Z)^{\prime} M(X)(\operatorname{vec} Z)$ $\geqslant \alpha$, for some $\alpha>0$, i.e., $(\operatorname{vec} Z)^{\prime}(-\alpha I+M(X))(\operatorname{vec} Z) \geqslant 0$. Hence, $X \in$ $\operatorname{SOL}_{\mathcal{B}}^{\text {Cone-LP }}(*,-\alpha I, M)$.

The interesting observation is that in fact the BMI has a solution if and only if Theorem 14 holds for any $\alpha>0$.

Corollary 15. The BMI has a solution if and only if for any $\alpha>0, S(\alpha) \cap \dot{\mathcal{B}} \neq \emptyset$.

Proof. Suppose there exist $\beta>0$ and $X \in \dot{\mathcal{B}}$ such that $-\beta I+M(X) \in \mathcal{B}^{*}$. Then for all $\alpha>0, \alpha X \in S(\alpha \beta)$, since $-\alpha \beta I+M(\alpha X)=\alpha(-\beta I+M(X)) \in \mathcal{B}^{*}$, and $\alpha X \in \dot{\mathcal{B}}$.

The implication of Corollary 15 is as follows: Given any $\alpha>0$, check whether $S(\alpha)$ contains an extreme form of the cone $\mathcal{B}$, which by Lemma 9 , has to be of rank one. This is the case if and only if the BMI has a solution. Geometrically, one can "attempt" to illustrate this implication as in Fig. 2.

The following conceptual algorithm is the result of our preceding discussion.

\section{A conceptual algorithm for solving the BMI}

1. Input the linear map $M$.

2. Choose $\alpha>0$; for example let $\alpha=1$.

3. Find the minimum rank solution $X \in \operatorname{SOL}_{\mathcal{B}}^{\text {Cone-LP }}(*,-\alpha I, M)$.

4. If $X \in \dot{\mathcal{B}}$, stop. $X \in \operatorname{SOL}_{\mathcal{B}}^{\mathrm{EFP}}(M)$, and therefore BMI has a solution.

5. If $X \notin \dot{\mathcal{B}}$, stop. The BMI does not have a solution. 


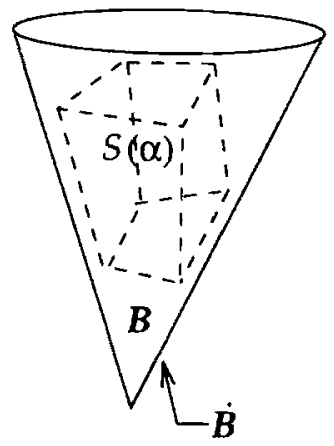

Fig. 2. BMI has a solution if and only if $S(\alpha) \cap \dot{B} \neq \emptyset$.

The main component of the above algorithm is Step 3. In this step, we are required to find the minimum rank element of a "matrix polyhedron" defined by the constraints $X \in \mathcal{B}$, and $-\alpha I+M(X) \in \mathcal{B}^{*}$. Clearly, this "matrix polyhedron" is a subset of the matrix cone $\mathcal{B}$, and is not necessarily "polyhedral."

Initial observations indicate that checking whether a "matrix polyhedron," over the cone of positive semi-definite matrices, contains a rank one element can be done efficiently for certain classes of linear maps [15]. On the other hand, the above Cone-LP approach calls for checking for the rank one element in a subset of the matrix cone $\mathcal{B}$. However, it is known that checking whether a matrix belongs to the cone of copositive matrices (for which $\mathcal{B}^{*} \equiv \mathcal{C}$ is a generalization of) is a difficult computational task [16]. Consequently, although the preceeding Cone-LP approach provides us with a way of understanding the geometry of the BMI, its computational realization runs into difficulty.

The above considerations have led us to adopt yet another approach for solving the BMI. The approach relies on establishing a connection between the BMI and a linear complementarity problem over the $\mathcal{P S D}$ cone (26). The main advantage of the complementarity approach is that one can formulate the resulting problem over a matrix cone for which an interior point algorithm can be developed. The complementarity approach also provides us with a way of addressing certain structural issues. This approach is examined next.

\subsection{The Cone-LCP formulation of the BMI}

In this section we explore the idea of viewing the BMI as a certain linear complementarity problem over a matrix cone (Cone-LCP). The EFP formulation of the BMI discussed in Section 3.3 is again the main tool for making the Cone-LCP approach possible.

Our motivation for the Cone-LCP approach is twofold. First, the complementarity approach enables one to address the two important structural issues (2) and (3) mentioned in Introduction. The basic idea is to use the rich theory that has been developed for the linear complementarity problems over the last few decades to examine the properties 
of the BMI. Additionally, we shall show that the Cone-LCP that arises from the BMI can be formulated on the $\mathcal{P S D}$ cone, rather than the less (computationally) understood matrix cone $\mathcal{B}$. The complementarity problem over the $\mathcal{P S D}$ cone also has the advantage of being readily amenable to an interior point approach [13]. However, one has to note that a "general" Cone-LCP is a difficult computational problem, as is the LCP itself. One of the main advantages of the Cone-LCP formulation however, is the ability of recognizing "efficiently" solvable instances.

In this section we shall assume that the Cone-LCP of the form Cone- $\operatorname{LCP}_{\mathcal{P S D}}(Q, M)$, with $M$ being a $\mathcal{P S D}$-copositive (see Section 2.1), can be solved in a "reasonable" time. This assumption is guided by the fact that a usual copositive LCP is a more "tractable" problem than a general LCP. Whether this approach yields a practical algorithm for the BMI depends heavily on the development of the efficient algorithms for the copositive Cone-LCPs, by using the interior point or other methods.

The starting point for the Cone-LCP approach is the observation made in Remark 12: the BMI has a solution if and only if the image of an extreme form of the matrix cone $\mathcal{P S D _ { 0 }}$ under the linear map $M$, is in the interior of $\mathcal{C}$, or in fact in $\mathcal{C}_{1}$, defined by $\mathcal{C}_{1}:=\operatorname{int} \mathcal{C} \cap \mathcal{C}_{0}$. As the next proposition states, the cone $\mathcal{P S D}$ can substitute the cone $\mathcal{P S D _ { 0 }}$ in the above statement.

Proposition 16. There exists an extreme form of the matrix cone $\mathcal{P S D}, X$, such that $M(X) \in \operatorname{int} \mathcal{C}$, if and only if there exists an extreme form of the matrix cone $\mathcal{P} S \mathcal{D}_{0}, X$, such that $M(Y) \in \operatorname{int} \mathcal{C}$.

Proof. An extreme form of the $\mathcal{P S D _ { 0 }}$ cone is also an extreme form of the $\mathcal{P S D}$ cone (Proposition 6). Therefore it suffices to show that if $X$ is an extreme form of the

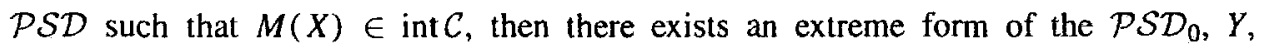
such that $M(Y) \in \operatorname{int} \mathcal{C}$. But this follows from the observation made in Section 3.1: If $X=(\operatorname{vec} W)(\operatorname{vec} W)^{\prime}, W=W_{1}+W_{2}$, with $W_{1} \in S \mathbb{R}^{p \times p}$ and $W_{2} \in \widehat{S} \mathbb{R}^{p \times p}$, let $Y=$ $\left(\operatorname{vec} W_{1}\right)\left(\operatorname{vec} W_{1}\right)^{\prime}$, and note that $M(X)=M(Y)$, since for $1 \leqslant i \leqslant n, H_{i} \operatorname{vec} W_{2}=0$ (refer to $(24)$ ).

Let us denote by $\bar{p}=p(p+1) / 2$ the dimension of the space of symmetric $p \times p$ matrices. Before stating the main result of this section we make the following observation.

Lemma 17. Let $Y$ be an extreme form of the cone $\mathcal{P S} \mathcal{D}_{0}$. Then there exists a symmetric $W \in \mathcal{P S} \mathcal{D}_{0}$, such that $Y \bullet W=0$ and $\operatorname{rank}(W)=\bar{p}-1$.

Proof. Let $Y=(\operatorname{vec} V)(\operatorname{vec} V)^{\prime}, V \in \mathbb{S}^{n \times p}$. Then there exists a set of linearly independent matrices $U_{i}$ 's $\in \mathrm{S}^{p \times p}(i=1, \ldots, \bar{p}-1)$, such that $U_{i} \bullet V=0$. Let $Z_{i}=$ $\left(\operatorname{vec} U_{i}\right)\left(\operatorname{vec} U_{i}\right)^{\prime}$. Then $Z_{i} \bullet Y=0, i=1, \ldots, \bar{p}-1$. Let $W=\sum_{i=1}^{\bar{p}-1} Z_{i}$. Then $W$ is symmetric, rank $(W)=\bar{p}-1$, and $W \in \mathcal{P S} \mathcal{D}_{0}$. 
Consider the Cone-LCP $\operatorname{PSD}_{\mathcal{D}}(Q, M)$ and let $M$ be defined by the equation (24): Find $X \in \mathrm{SR}^{n^{2} \times p^{2}}$ (if it exists) such that:

$$
\begin{aligned}
& X \in \mathcal{P} \mathcal{S D}, \\
& Q+M(X) \in \mathcal{P} \mathcal{S D}^{*} \equiv \mathcal{P} \mathcal{S D}, \\
& X \bullet(Q+M(X))=0
\end{aligned}
$$

The main result of this section now follows.

Theorem 18. The BMI has a solution if and only if there exists a matrix $Q \in$ int $(-\mathcal{C})$ (or $\left.Q \in-\mathcal{C}_{1}\right)$, such that the $\operatorname{Cone-\operatorname {LCP}} \operatorname{PSD}_{\mathcal{S}}(Q, M)$ has a rank one solution.

Proof. (Necessity) Suppose the BMI has a solution $X^{*}$, that is, $X^{*}=(\operatorname{vec} Y)(\operatorname{vec} Y)^{\prime}$, $Y \in S \mathbb{R}^{p \times p}, M\left(X^{*}\right) \in \operatorname{int} \mathcal{C}$, and in fact $M\left(X^{*}\right) \in \mathcal{C}_{1}$. By Lemma 17 , there exists $W \in \mathcal{P S D _ { 0 }}$, rank $W=\bar{p}-1$, such that $W \bullet X^{*}=0$. Without loss of generality, assume that $\|W\|=1$.

Let $Q_{\alpha}=\alpha W-M\left(X^{*}\right)$. Note that since $M(X)$ is symmetric (for $X \in \mathcal{P S} \mathcal{D}_{0}$ ), $Q_{\alpha}$ is also symmetric. Moreover, $Q_{\alpha}(\operatorname{vec} Z)=0$, for all $Z \in \widehat{S} \mathbb{R}^{p \times p}$, since both $M(X)$ and $W$ are in the $\mathcal{P} \mathcal{S D}_{0}$ ( see Eq. (25)). It suffices to show that there exists an $\alpha>0$, such that $Q_{\alpha} \in \operatorname{int}\left(-\mathcal{C}\right.$ ) (or $Q \in-\mathcal{C}_{1}$ ). Since $M\left(X^{*}\right) \in \operatorname{int} \mathcal{C}$ and $\mathcal{B}$ is closed, there exists $\beta>0$, such that inf $\operatorname{loB}_{U \in \mathcal{B}, \|=1} U \bullet M\left(X^{*}\right) \geqslant \beta>0$.

Hence, for all $U \in \mathcal{B},\|U\|=1$,

$$
\begin{aligned}
U \bullet Q_{\alpha} & =U \bullet\left(\alpha W-M\left(X^{*}\right)\right)=\alpha(U \bullet W)-U \bullet M\left(X^{*}\right) \\
& \leqslant \alpha U \bullet W-\beta \leqslant \alpha-\beta .
\end{aligned}
$$

Therefore choosing $\bar{\alpha}<\beta$, we see that for all $U \in \mathcal{B},\|U\|=1, U \bullet Q_{\bar{\alpha}}<0$. Hence $Q_{\bar{\alpha}} \in \operatorname{int}\left(-\mathcal{C}\right.$ ) (in fact $Q_{\bar{\alpha}} \in-\mathcal{C}_{1}$ ).

Moreover,

$$
0=\bar{\alpha}\left(X^{*} \bullet W\right)=X^{*} \bullet\left(Q_{\bar{\alpha}}+M\left(X^{*}\right)\right) .
$$

By construction, $X^{*} \in \mathcal{P S D}$, rank $X^{*}=1$, and $\left(Q_{\bar{\alpha}}+M\left(X^{*}\right)\right) \in \mathcal{P S D} \mathcal{D}_{0} \subseteq \mathcal{P S D}$.

(Sufficiency) Suppose there exists a rank one matrix $X^{*}, X^{*} \in \operatorname{SOL}_{\mathcal{P} S D}^{\text {Cone-LCP }}(Q, M)$, with $Q \in \operatorname{int}(-\mathcal{C})$. Then there exists $Z^{*} \in \mathbb{R}^{p \times p}$ such that,

$$
X^{*}=\left(\operatorname{vec} Z^{*}\right)\left(\operatorname{vec} Z^{*}\right)^{\prime} \in \mathcal{P S D} \text {. }
$$

Since $Q \in \operatorname{int}(-\mathcal{C})$ and $Q+M\left(X^{*}\right) \in \mathcal{P S D}$, using the inclusion $\mathcal{B} \subseteq \mathcal{P S D}$, one has the following:

$$
\begin{aligned}
\forall A \in \mathcal{B}(A \neq 0): A \bullet M\left(X^{*}\right) & =A \bullet\left(Q+M\left(X^{*}\right)-Q\right) \\
& =A \bullet\left(Q+M\left(X^{*}\right)\right)-A \bullet Q>0 .
\end{aligned}
$$

The last inequality follows from the fact that, for all $A \in \mathcal{B}(A \neq 0), A \bullet Q<0$. Consequently, $M\left(X^{*}\right) \in \operatorname{int} \mathcal{C}$. 
In view of Proposition 16, there exists a rank one matrix,

$$
Y^{*}=\left(\operatorname{vec} W^{*}\right)\left(\operatorname{vec} W^{*}\right)^{\prime} \in \mathcal{P} \mathcal{S} \mathcal{D}_{0}, \quad W \in S \mathbb{R}^{p \times p}
$$

such that $M\left(Y^{*}\right) \in \operatorname{int} \mathcal{C}$. Therefore the BMI has a solution.

The above proof can be modified in an obvious way to conclude that it is only sufficient to take $Q \in-\mathcal{C}_{1}$.

We shall refer to the special case of the linear complementarity problem over the positive semi-definite cone (34)-(36), as the Semi-Definite Complementarity Problem (SDCP). An immediate consequence of the above theorem is that if a matrix $Q \in$ $\operatorname{int}(-\mathcal{C})$ cannot be found for which the corresponding SDCP has a solution, then the $\mathrm{BMI}$ does not have a solution.

Corollary 19. The BMI does not have a solution if the SDCP (34)-(36) is not solvable for any $Q \in \operatorname{int}(-\mathcal{C})$ (or in fact $Q \in-\mathcal{C}_{1}$ ).

It is noteworthy that the linear map $M$ in the SDCP formulation, which arises in the context of the BMI, is itself copositive with respect to the matrix cone $\mathcal{P S D}$.

Proposition 20. The linear map $M$ defined by (24) is $\mathcal{P S D}$-copositive. Consequently, if we define $M^{*}(X)=\sum_{i=1}^{n} H_{i}^{\prime} X H_{i}$, and the implication

$$
X \in \mathcal{P S D}, X \bullet M(X)=0 \Rightarrow M(X)+M^{*}(X)=0
$$

holds, then for all $Q \in-\mathcal{C}_{1}$, the Cone-LCPPSD $(Q, M)$ is solvable if it is feasible.

Proof. Note that for all $X \in \mathcal{P S D}, M(X) \in \mathcal{P S D}$ due to the special structure of the linear map $M$ (see Eq,(25)). Since $\mathcal{P S D} \mathcal{D}^{*}=\mathcal{P S D}, X \bullet M(X) \geqslant 0$, for all $X \in \mathcal{P} S D$. If the implication (37) holds then $M(X)$ is indeed a $\mathcal{P S D}$-copositive plus map (which basically means that $M$ is $\mathcal{P S D}$-copositive and that the implication (37) holds). We now elaborate on the solvability of the feasible copositive-plus Cone-LCP $\operatorname{PSD}_{\mathcal{D}}(Q, M)$, for all $Q \in-\mathcal{C}_{1}$, based on the result of Gowda and Seidman [7]. According to Theorem 4.1 of [7], if for a convex cone $\mathcal{K}$ in a finite-dimensional Hilbert space the condition,

$$
\left\{X \in \mathcal{K} \mid M(X) \in \mathcal{K}^{*},\langle M(X), X\rangle=0,<Q, X>=0\right\}=\{0\}
$$

holds and $M$ is copositive plus on $\mathcal{K}$, then the solution set of the corresponding Cone-LCP $\operatorname{LP}_{\mathcal{K}}(Q, M)$ is non-empty. The statement of the proposition now follows by observing that for all $Q \in-\mathcal{C}_{1} \subseteq-$ int $\mathcal{C}$, the only $X \in \mathcal{P S D} \subseteq \mathcal{C}$ for which $Q \bullet X=0$ is $X=0$.

If one assumes that the copositive SDCPs can be solved efficiently, then the above proposition implies that the SDCP which arises from the BMI can be solved efficiently for each $Q$. Moreover, if for a particular $M$, implication (37) holds, then the corresponding SDCP is always solvable, if the BMI has a solution (using Proposition 10). 
If the BMI has a solution, then knowledge of the direction of $Q$ in the corresponding Cone-LCP $\operatorname{PPD}_{\mathcal{S}}(Q, M)$ is sufficient for finding the solution of the BMI. In other words, if this direction is known, the solution set of a single SDCP should be examined. This result is established by the following corollary.

Corollary 21. The BMI has a solution if and only if there exists a symmetric $Q \in$

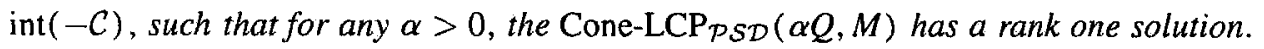

Proof. Using the result of Theorem 18, the proof is similar to the one given for Corollary 15 , and is therefore omitted.

In general, finding the direction of the matrix $Q \in \operatorname{int}(-\mathcal{C})$ in Theorem 18 is not trivial. In the case where an additional assumption on the solution set of the $\operatorname{EFP}_{\mathcal{B}}(M)$ is made, the direction can be chosen a priori.

Corollary 22. Suppose that for all $X^{*} \in \operatorname{SOL}_{\mathcal{B}}^{\mathrm{EFP}}(M)$,

$$
\begin{aligned}
& X^{*}=\left(\operatorname{vec} U^{*}\right)\left(\operatorname{vec} U^{*}\right)^{\prime}, \quad U^{*} \succeq 0,\left\|\operatorname{vec} U^{*}\right\|=1, \\
& X^{*} \bullet M\left(X^{*}\right) \leqslant Y \bullet M\left(X^{*}\right)
\end{aligned}
$$

for all extreme forms $Y$ of $\mathcal{B}\left(Y \neq X^{*}\right), Y=(\operatorname{vec} V)(\operatorname{vec} V)^{\prime},\|\operatorname{vec} V\|=1$. Then the matrix $Q \in$ int $(-\mathcal{C})$ in Theorem 18 can be taken to be any positive multiple of $-I_{p^{2} \times p^{2}}$.

Proof. First note that $X^{*} \cdot I=\left(\operatorname{vec} U^{*}\right)^{\prime}\left(\operatorname{vec} U^{*}\right)=\left\|\operatorname{vec} U^{*}\right\|^{2}=1$. Similarly, for all $Y$ of $\mathcal{B}, Y=(\operatorname{vec} V)(\operatorname{vec} V)^{\prime},\|\operatorname{vec} V\|=1, Y \bullet I=1$. Since $M\left(X^{*}\right) \in \operatorname{int} \mathcal{C}$, there exists $\alpha>0$, such that $X^{*} \bullet\left(-\alpha I+M\left(X^{*}\right)\right)=0$. To guarantee that $-\alpha I+M(X) \in \mathcal{C}$, we require that for all $Z \in \mathcal{B}, Z \bullet(-\alpha I+M(X)) \geqslant 0$. But this is implied by the assumption of the corollary, since if (38) holds, for all extreme forms $Y \neq X, Y=(\operatorname{vec} V)(\operatorname{vec} V)^{\prime}$, $\|\operatorname{vec} V\|=1, Y \bullet\left(-\alpha I+M\left(X^{*}\right)\right) \geqslant X^{*} \bullet\left(-\alpha I+M\left(X^{*}\right)\right)=0$. Using Corollary 21, the result of the corollary now follows.

Remark 23. If in the above corollary one prefers to find a direction in $-\mathcal{C}_{\mathrm{I}}$, then all the norm of vec conditions should be replaced by the trace condition, and $-I_{p^{2} \times p^{2}}$ by $-\left(\operatorname{vec} I_{p \times p}\right)\left(\operatorname{vec} I_{p \times p}\right)^{\prime} \in-\mathcal{C}_{\mathrm{I}}$. The statement of the corollary would still be valid with these modifications.

Corollary 22 reduces the BMI to examining the solution set of an SDCP with a $\mathcal{P S D}$-copositive linear map. In fact, the $Q$ matrices that arise in this context have a very special form.

The copositive SDCPs have much more structure than a general SDCP. Nevertheless, it remains to be shown whether this class of complementarity problems can be solved efficiently. The generalizations of the Lemke's algorithm for solving the copositive LCPs, or the interior point methods for the Semi-Definite Programming Problem (SDP), are possible objectives that could be pursued for this purpose. 
Another Cone-LCP formulation of the BMI, besides the one mentioned above, is to incorporate the problem of finding the matrix $Q$ in Theorem 18 , in setting up the corresponding Cone-LCP. For this purpose it is convenient to associate to the matrix cones $\mathcal{P S D}, \mathcal{B}$, and $\mathcal{C}$ (subsets of $S \mathbb{R}^{p^{2} \times p^{2}}$ ), the cones $\overline{\mathcal{P S D}}, \overline{\mathcal{B}}$, and $\overline{\mathcal{C}}$ (subsets of $\mathbb{R}^{r^{4}}$ ), which are obtained by applying the vec operator to these matrix cones, i.e.,

$$
\overline{\mathcal{P S D}}=\left\{x \in \mathbb{R}^{\prime^{\prime \prime}} \mid x=\operatorname{vec} A, A \in \mathcal{P S D}\right\}
$$

and

$$
\overline{\mathcal{B}}=\left\{x \in \mathbb{R}^{p^{4}} \mid x=\operatorname{vec} A, A \in \mathcal{B}\right\}, \quad \overline{\mathcal{C}}=\left\{x \in \mathbb{R}^{p^{4}} \mid x=\operatorname{vec} A, A \in \mathcal{C}\right\} .
$$

It is easy to verify that $\overline{\mathcal{P S D}}, \overline{\mathcal{B}}$, and $\overline{\mathcal{C}}$ are closed convex cones in $\mathbb{R}^{P^{4}}$.

Recall that for all $A, B \in \mathrm{S}_{\mathbb{R}^{p^{2}} \times p^{2}}$,

$$
A \cdot B \geqslant 0 \Longleftrightarrow(\operatorname{vec} A)^{\prime}(\operatorname{vec} B) \geqslant 0 \text {. }
$$

Therefore, in view of the relation $\mathcal{P S D}=\mathcal{P S D}$, the only matrices in $\overline{\mathcal{P S D}}^{*}$ that are the vec form of a symmetric matrix, are those in $\overline{\mathcal{P S}} \overline{\mathcal{D}}$.

Let $H=\sum_{i=1}^{n} H_{i} \otimes H_{i} \in \mathbb{R}^{p^{4} \times p^{4}}$. For the linear map $M$ defined by (24) and using the property of the Kronecker products, vec $M(X)=H \operatorname{vec} X$. Combining the above ideas with the result of Theorem 18, one readily obtains the following corollary.

Corollary 24. Let

$$
\widetilde{M}=\left(\begin{array}{cc}
0 & 0 \\
-I_{p^{4}} \times p^{4} & H
\end{array}\right)
$$

and,

$$
Z=\left(\begin{array}{c}
\operatorname{vec}-Q \\
\operatorname{vec} X
\end{array}\right) \in \overline{\mathcal{C}} \times \overline{\mathcal{P S D}}
$$

Then the BMI has a solution if and only if the homogeneous Cone- $\operatorname{LCP}_{\overline{\mathcal{C}} \times \overline{\mathcal{P S D}}}(0, \tilde{M})$ has a solution of the form

$$
\widetilde{Z}=\left(\begin{array}{c}
\operatorname{vec}-\widetilde{Q} \\
\operatorname{vec} \widetilde{X}
\end{array}\right)
$$

where $-\tilde{Q} \in$ int $\mathcal{C}$, and $\widetilde{X}$ has rank one.

We note that if $Q \in-\mathcal{C}$ and $X \in \mathcal{P S D}$, then $Q+M(X)$ is automatically symmetric and therefore if $\operatorname{vec}\left(Q+M\left(X^{*}\right)\right) \in \overline{\mathcal{P S D}}^{*}$, then $Q+M\left(X^{*}\right) \in \mathcal{P} \mathcal{S D}$.

The above corollary reduces the BMI feasibility problem to the problem of examining the solution set of a certain Cone-LCP. This can be a "tractable" problem if the solution set is finite, or if the linear map $\widetilde{M}$ enjoys certain "additional" properties. Since there are various results in the complementarity theory which pertain to the cardinality of the solution set of a Cone-LCP [11], classification of efficiently solvable instances of the BMI can be based on those results as well. 


\section{Concluding remarks}

In this paper, we have established various connections between the Bilinear Matrix Inequality (BMI) and three problems over matrix cones. The first two problems, which we have referred to as the Extreme Form Problem (EFP) and the linear programming problem over matrix cones (Cone-LP), were formulated over a generalization of the cone of completely positive matrices. The above two cone problems facilitate our understanding of the geometry of the BMI. Nevertheless, the computational implications of these formulations run into difficulty, since the completely positive matrices can not be efficiently characterized by means of an algorithm. The last cone problem which we have established its connection with the BMI, is the linear complementarity problem over the cone of positive semi-definite matrices (SDCP). The SDCP is readily amenable to an interior point approach. In this later case, the existence of a solution to the BMI is checked by examining the solution set of an SDCP. The actual solution to the BMI can then be constructed by solving a linear matrix inequality (LMI) which can be done via an interior point algorithm.

This work can be continued in several directions. One such direction is to study in greater detail, the properties of the various matrix cones introduced in this paper. We believe that the Extreme Form Problem (EFP) and the linear complementarity problem over the positive semi-definite matrices (SDCP) are of independent interest, which warrants research efforts for understanding of their properties and constructing computational procedures for their solution.

\section{Acknowledgment}

We gratefully acknowledge fruitful discussions with N. Fathpour, K.C. Goh, M.G. Safonov, F. Alizadeh, M. Ramana, and thank the two anonymous referees for their very valuable suggestions and corrections. In particular, the papers of Gowda [6], Gowda and Seidman [7], and Kojima et al. [12], were brought to our attention by the referees.

\section{References}

11] F. Alizadeh, Interior point methods in semi-definite programming with applications to combinatorial optimization, SIAM Journal of Optimization 5 ( I) (1995) 13-51.

121 A. Berman, Cones, Matrices, and Mathematical Programming (Springer, Berlin, 1973).

13| S.P. Boyd, L. El Ghaoui, E. Feron and V. Balakrishnan, Linear Matrix Inequalities in System and Control Theory (SIAM, Philadelphia, PA, 1994).

14| R.W. Cottle, J.S. Pang and R.E. Stone, The Linear Complementarity Problem (Academic Press, Boston, MA, 1992).

[5] K.C. Goh, M.G. Safonov and G.P. Papavassilopoulos, A global optimization approach for the BMI problem, in: Proceedings of the IEEE Conference on Decision and Control, Lake Buena Vista, FL, 1994.

[6] M.S. Gowda, Complementary problems over locally compact cones, SIAM Joumal of Control and Optimization 27 (4) (1989) 836-841. 
|7| M.S. Gowda and T.I. Seidman, Generalized linear complementarity problems, Mashematical Programming 46 (1990) 329-340.

|8| M. Hall Jr, Combinatorial Theory (Blaisdell Publishing Company, Waltham, MA. 1967).

19] R.A. Horn and C.R. Johnson, Marrix Analysis (Cambridge University Press, Cambridge, MA, 1985).

[10] R.A. Horn and C.R. Johnson, Topics in Marrix Analysis (Cambridge University Press, Cambridge, MA, 1991).

|11] G. Isac, Complementarity Problems (Springer, Berlin, 1992).

112| M. Kojima, M. Shida and S. Shindoh, Reduction of linear complementarity problems over cones to linear programming over cones, Technical Report, Department of Information Science, Tokyo Institute of Technology, Japan, 1995.

I. 13| M. Kojima, S. Shindoh and S. Hara, Interior-point methods for the monotone linear complementarity problem in symmetric matrices, Technical Report, Department of Information Science, Tokyo Institute of Technology, Japan, 1994.

[14] M.G. Krein and M.A. Rutman, Linear operators leaving invariant a cone in a Banach space, American Mathematical Society Translations 1 (10) (1956) 199-325.

[15] M. Mesbahi and G.P. Papavassilopoulos. On the rank minimization problem over a positive semidefinite linear matrix inequality, IEEE Transactions on Automatic Comtrol 42 (2) (1997) 239-243.

[16] K.G. Murty and S.N. Kabadi, Some NP-complete problems in quadratic and nonlinear programming, Mathemarical Programming 39 (1987) 117-129.

| $17 \mid$ Y. Nesterov and A. Nemirovskii, Interior-Point Polynomial Algorithms in Convex Programming (SIAM, Philadelphia, PA, 1994).

[18] R.T. Rockafellar, Convex Analysis (Princeton University Press. Princeton, NJ, 1970).

[19] M.G. Safonov, K.C. Goh and J.H. Ly, Control system synthesis via bilinear matrix inequalities, in: Proceedings of the 1994 American Control Conference, Baltimore. MD, 1994.

[20] M.G. Safonov and G.P. Papavassilopoulos, The diameter of an intersection of ellipsoids and BMI robust synthesis, in: IFAC Symposium on Robust Control, Rio de Janeiro, Brazil, 1994.

[21] J. Stoer and C. Witzgall, Convexity and Optimization in Finite Dimension (Springer, Berlin, 1970).

[22] O. Toker and $\mathrm{H}$. Özbay, On the NP-hardness of solving bilinear matrix inequalities and simultaneous stabilization with static output feedback, in: Proceedings of the 1995 American Control Conference, Seattle, Washington, 1995. 